\title{
FREE VIBRATION ANALYSIS OF POINT SUPPORTED RECTANGULAR PLATES USING QUADRATURE ELEMENT METHOD
}

\author{
Ahmet Çetkin \\ Afyon Kocatepe University, Technical Education Faculty, Afyon, Turkey \\ e-mail: acetkin@aku.edu.tr \\ S. ORAK \\ Osmangazi University, Department of Mechanical Engineering, Eskisehir, Turkey \\ e-mail:sorak@ogu.edu.tr
}

\begin{abstract}
In this study, the hybrid approach of the Quadrature Element Method (QEM) has been employed to generate solutions for point supported isotropic plates. The Hybrid QEM technique consists of a collocation method with the Galerkin finite element technique to combine the high accurate and rapid converging of Differential Quadrature Method (DQM) for efficient solution of differential equations. To present the validity of the solutions, the results have been compared with other known solutions for point supported rectangular plates. In addition, different solutions are carried out for different type boundary conditions, different locations and number of point supports. Results for the first vibration modes of plates are also tested using a commercial finite element code, and it is shown that they are in good agreement with literature.
\end{abstract}

Keywords: Quadrature Element Method, point support, plates, free vibration

\section{Introduction}

In the applications of modern structures, i.e. carousers, building floors, bridge decks, solar panels, aircraft and ship industries, bolted, riveted or spot-welded plate bodies are used. Designers have to know how these components change the dynamic characteristic of the structures. These types of engineering problems are known as point supported plate problems and they are frequently encountered in practice. Both analytical and numerical methods have been developed for the analysis of these problems. Although there are no exact solutions for these problems, various numerical approaches have been utilized. For example, Cox and Boxer (1960) used a finite difference method, Damle and Feeser (1972) used the finite element method, Fan and Cheung (1984) used the spline finite strip method, Huang and Thambiratnam (2001a) used the finite strip method, Guiterrez and Laura (1995) used dthe ifferential quadrature method, Zhao et al. (2002) used the discrete singular convolution method to solve the mentioned plate vibration problems. Because of its high accuracy, the Rayleight-Ritz method has been the most frequently used analytical method to appeal for vibration analysis of plates, as Narita and Hodgkinson (2005) did. Also Gorman (1991) and Bapat and Suryanarayan (1989) utilized the superposition method and the flexibility function approach as analytical techniques, respectively.

Several functions are used for the analysis of free vibration of point supported rectangular plates. These include vibrating beam functions (Kerstens, 1979), B-spline functions (Mizusawa and Kajita, 1987)] and orthogonal polynomial functions (Kim and Dickinson, 1987). On the other hand, Liew and Lam (1994) applied a set of orthogonal plate functions generated by using the Gram-Schmidt orthogonality relationship to elastic point supported rectangular plates. Lee and Lee (1997) used a new type of the admissible function. Kitipornchai et al. (1994) and Liew et al. (1994) applied the Lagrange multiplier method and the constrain function method to 
point supported Mindlin plates. Cheung and Zhou (1999, 2000) used the static beam function to composite plates and used the finite layer method to layered rectangular plates with point supports. Saadatpoure et al. (2000) studied vibration of plates having a general shape with internal point and line supports using the Galerkin method. Huang and Thambiratnam (2001b) applied a procedure incorporating the finite strip method together with spring systems for treating plates on elastic intermediate supports. Zhou (2002) used a set of static tapered beam functions which were the solutions of a tapered beam under a Taylor series of static loads developed as admissible functions for vibration analysis of point-supported rectangular plates with variable thickness in one or two directions. Again, Zhao et al. (2002) studied the problem of plate vibration under complex and irregular internal support conditions using the discrete singular convolution method. Kocatürk et al. (2004) used Lagrange equations to examine the steady state response to a sinusoidally varying force applied at the centre of a viscoelastically point-supported orthotropic elastic plate of rectangular shape with considered locations of added masses.

The Differential Quadrature Method (DQM) was proposed by Bellman and Casti (1971) in the early 1970's as an efficient numerical method to solve non-linear partial differential equations and applied to many areas of engineering problems. Especially, the Generalized Differential Quadrature Method (GDQM) has been used by various researches for efficient treatment of structural analysis problems. Analyses yielded good to excellent results for only a few discrete points due to the use of high order global basis functions in the computational domain. However, especially for real-world problems, DQM still lacks flexibility. Recently, Chen et al. (2000) extended the DQM to analysis of various structures and then it called the Quadrature Element Method (QEM). 49 degree of freedom (DOF) quadrature plate element was developed by Striz et al. (1994) to alleviate the lack of versatility and limitations of the existing high order series type approximation method. Different versions of the Differential Quadrature Method have been used for various applications. Hybrid approach was further developed by Han and Liew (1996) to solve the one-dimensional bending problem of the axisymmetric shear deformable circular plate, and by Liu and Liew (1998, 1999a,b) and Liu (2000) to solve two-dimensional bending and vibration problems of thick rectangular plates and polar plates having discontinuities. Wang and $\mathrm{Gu}(1997 \mathrm{a}, \mathrm{b})$ made an attempt to solve static problems of truss and beams and static and free vibration problems of thin plates. DQM was used by Liu and Liew (1999b) for the study of a two dimensional polar Reissner-Mindlin plate in the polar coordinate system by integrating the domain decomposition method (DDM). The Differential Quadrature Finite Difference Method (DQFDM) was proposed and applied by Chen (2004) for analysis of 2-D heat conduction in orthotropic media. Franciosi and Tomasiello (2004) applied a modified quadrature element method to perform static analysis of structures.

In this paper, the Quadrature Element Method is proposed and applied to analyze free vibration of point supported rectangular plates. Plates having different boundary conditions and various point topologies are studied. The results are compared with the studies using other approximating methods known in literature. First, interior and/or exterior point supported free plates and then, interior point supported plates having various boundary conditions are presented. Solutions are tested with the results of ABAQUS, a finite element program which has a wide spread use in the analysis of engineering problems.

\section{Formulation of the quadrature plate element}

The Hybrid Quadrature Element technique consists of a collocation method in conjunction with the Galerkin finite element technique to combines the high accuracy and rapid converging of DQM for efficient solution of differential equations with the generality of the finite element formulation (Chen et al., 2000). 
The quadrature plate element is closely related to the serendipity Lagragian element, but it has internal points and basis functions of high order (Chen et al., 2000). Numerical procedures are extensively used in the element formulation to circumvent the problems caused by the use of high order basis functions. $C_{0}$ and $C_{1}$ inter-element compabilities are met exactly for the mid-surface, while the other $C_{2}$ or even $C_{3}$ compabilities are closely approximated at each boundary by the use of moderately high order basis functions. The 25 node rectangular element is given in Fig. 1. This plate element has also 49 degrees of freedom. These degrees of freedom, which belong to the plate element, are given in Table 1 (Chen et al., 2000; Quan and Chang, 1989).

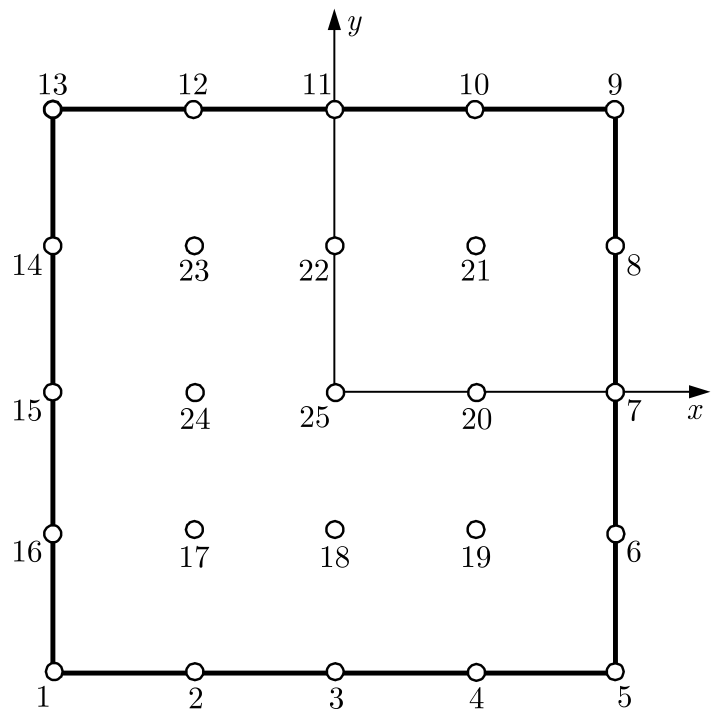

Fig. 1. Nodes of the Quadrature plate element

Table 1. Degrees of freedom for 25 node quadrature plate elements

\begin{tabular}{|c|c|}
\hline Nodal number & DOF \\
\hline \hline $1-5$ & $w, \frac{\partial w}{\partial x}, \frac{\partial w}{\partial y}, \frac{\partial^{2} w}{\partial x \partial y}$ \\
\hline $9-13$ & $w, \frac{\partial w}{\partial y}$ \\
\hline $2-3-4$ & $w, \frac{\partial w}{\partial x}$ \\
\hline $10-11-12$ & \\
$6-7-8$ & \\
$14-15-16$ & \\
\hline $17-18-19$ & \\
$20-21-22$ & \\
$23-24-25$ & \\
\hline
\end{tabular}

The displacements of 25 nodes and 49 degrees of the freedom quadrature plate element are expressed in terms of polynomial type basis functions, i.e.

$$
\begin{aligned}
& w(x, y)=\sum_{i=1,5,9,13}\left[N_{i 1} w_{i}+N_{i 2}\left(\frac{\partial w}{\partial x}\right)_{i}+N_{i 3}\left(\frac{\partial w}{\partial y}\right)_{i}+N_{i 4}\left(\frac{\partial^{2} w}{\partial x \partial y}\right)_{i}\right] \\
& +\sum_{i=2,3,4,10,11,12}\left[N_{i 1} w_{i}+N_{i 2}\left(\frac{\partial w}{\partial y}\right)_{i}\right]+\sum_{i=6,7,8,14,15,16}\left[N_{i 1} w_{i}+N_{i 2}\left(\frac{\partial w}{\partial x}\right)_{i}\right] \\
& +\sum_{\substack{i=17,18,19,20 \\
21,22,23,24,25}}\left[N_{i 1} w_{i}\right]=\mathbf{N w}
\end{aligned}
$$


where $N_{i j}$ is the shape function which can be determined from the specified collocation points, and $w_{i},(\partial w / \partial x)_{i},(\partial w / \partial y)_{i},\left(\partial^{2} w / \partial x \partial y\right)_{i}$ are local DOFs associated with the node $i$. by

The governing equation of the isotropic thin plate in small deflection free vibration is given

$$
\frac{\partial^{4} w}{\partial x^{4}}+2 \frac{\partial^{4} w}{\partial x^{2} \partial y^{2}}+\frac{\partial^{4} w}{\partial y^{4}}=\frac{\rho h \omega^{2}}{D} w
$$

and Kirchhoff's plate theory, in which the bending strain of the element is given for an isotropic and homogeneous plate as

$$
\varepsilon=\left\{\begin{array}{c}
\varepsilon_{x} \\
\varepsilon_{y} \\
\gamma_{x y}
\end{array}\right\}=-z\left\{\begin{array}{c}
\frac{\partial^{2} w}{\partial x^{2}} \\
\frac{\partial^{2} w}{\partial y^{2}} \\
2 \frac{\partial^{2} w}{\partial x \partial y}
\end{array}\right\}
$$

If Eq. (2.1) and Eq. (2.3) are combined, the strain-displacement relationship is stated by

$$
\boldsymbol{\varepsilon}=-z\left\{\begin{array}{c}
\frac{\partial^{2} w}{\partial x^{2}} \\
\frac{\partial^{2} w}{\partial y^{2}} \\
2 \frac{\partial^{2} w}{\partial x \partial y}
\end{array}\right\} \mathbf{N w}=-z \mathbf{Q w} \quad \text { for } \quad \mathbf{Q}=\left\{\begin{array}{c}
\frac{\partial^{2} w}{\partial x^{2}} \\
\frac{\partial^{2} w}{\partial y^{2}} \\
2 \frac{\partial^{2} w}{\partial x \partial y}
\end{array}\right\} \mathbf{N}
$$

The stiffness matrix can be calculated for the area $A$

$$
\mathbf{K}=\int_{A} \mathbf{Q}^{\mathrm{T}} \mathbf{D Q} d A
$$

where $\mathbf{D}$ is the rigidity matrix which can be calculated using constant thickness $h$, Poisson's ratio $\nu$ and the modulus of elasticity $E$

$$
\mathbf{D}=\frac{E h^{2}}{12\left(1-\nu^{2}\right)}\left[\begin{array}{ccc}
1 & \nu & 0 \\
\nu & 1 & 0 \\
0 & 0 & (1-\nu) / 2
\end{array}\right]
$$

The consistent mass matrix can be calculated as

$$
\mathbf{M}=\int_{A} \mathbf{N}^{\mathrm{T}}(\rho \mathbf{h}) \mathbf{N} d A
$$

and the governing equation for plate free vibration can be written in the matrix form

$$
\left(\mathbf{K}_{s}-\lambda^{2} \mathbf{M}_{s}\right) \mathbf{w}=\mathbf{0}
$$

where $\lambda$ is the frequency parameter, and the subscribed $s$ represents the whole discretized system.

\section{Numerical application and discussions}

Frequency parameters of free vibrations are described as $\lambda=\omega L^{2} \sqrt{\rho h / D}$, where $\omega, L, \rho, h$, $D$ represent circular frequency, length of the plate, density, thickness and rigidity, respectively. 
In order to obtain more accurate results, QEM solutions have been carried out by using $2 \times 2$ and $4 \times 4$ differential quadrate plate elements joined side by side along the $x$ and $y$ directions. When a larger number of plate elements are used more accurate results can be obtained, but the solution can be obtained with a larger linear system of equations. If there are simply supported boundary conditions on all edges of the plate considered then the quadrate plate element has only 25 DOFs. In other words, a set of $25 \times 25$ linear equations system has to be solved for one plate element. The size of the linear equations system is set to $400 \times 400$ for the same procedure needed be to solve with the same boundary conditions and the $4 \times 4$ plate element.

First, the number of plate elements that can be used for results having acceptable accuracy must be decided. Therefore, frequency parameters for three boundary conditions and four plate elements are obtained with QEM. Table 2 presents the frequency parameters $\lambda$ of isotropic rectangular plates. It is interesting that acceptable accuracy results are obtained by QEM for all boundary conditions in the case of only one plate element.

Table 2. The first frequency parameters $\lambda$ of isotropic square plates for some boundary conditions $\left(\lambda=\omega L^{2} \sqrt{\rho h / D}\right)$

\begin{tabular}{|c|c|c|c|c|c|}
\hline & \multirow{2}{*}{$\begin{array}{c}\text { Exact } \\
\text { Leissa, 1973) }\end{array}$} & \multicolumn{4}{|c|}{ Number of use DQ plate elements } \\
\cline { 3 - 6 } & \multirow{2}{*}{19.73921} & $\begin{array}{c}19.73921 \\
\left(7.0 \cdot 10^{-4}\right)^{*}\end{array}$ & $\begin{array}{c}19.73921 \\
\left(2.2 \cdot 10^{-5}\right)^{*}\end{array}$ & $\begin{array}{c}19.73921 \\
\left(4.3 \cdot 10^{-7}\right)^{*}\end{array}$ & $\begin{array}{c}19.73921 \\
\left(1.6 \cdot 10^{-8}\right)^{*}\end{array}$ \\
\hline \hline S-S-S-S & & 9.63139 & 9.63138 & 9.63138 & 9.63138 \\
\hline S-F-S-F & 9.63138 & 23.64700 & 23.64632 & 23.64632 & 23.64632 \\
\hline S-C-S-S & 23.64632 & 232 &
\end{tabular}

* Relative error in parenthesis has been evaluated using the analytical Leissa value $\left(2 \pi^{2}\right)[\%]$

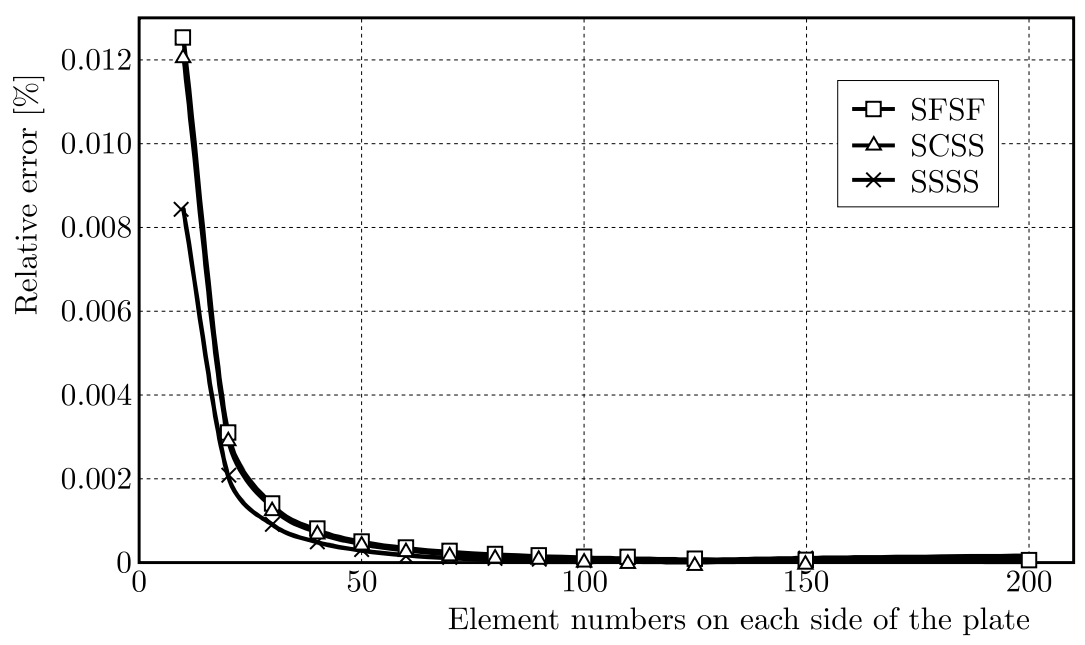

Fig. 2. Relative error determined by ABAQUS for different boundary conditions

Besides, the same boundary conditions given in Table 2 are solved using ABAQUS commercial finite codes. It is obvious that if more elements are used in computation, the error will be reduced. However, the required number of elements must be determined for acceptable accuracy. The variation of the relative error with selected degrees of freedom is given in Fig. 2 for different boundary conditions. Relative errors have been evaluated using the analytical results of Leissa (1973). This \% error value of the relative difference is defined as (Analytical Leissa value-ABAQUS result) $\times 100 /($ Analytical Leissa value). Naturally, the result changes when different boundary conditions are used. As in many literature sources the 4 node thin shell elements (S4R) are employed, the uniform mesh size and different element numbers on each side of the plate such as 10, 20, 50, 100, 200 and 400 scales are used to achieve convergent FEM solutions 
(Rui et al., 2015, 2016). In this study, the results have been given for all values from 100 SR4 shell elements on each side of the plate. For these elements, there are approximately 49,800 DOFs. As shown in Fig. 2, the biggest \% relative error for SFSF boundary conditions to the selected number of elements is $0.01 \%$.

In order to simplify the visualisation of types of supports which are used in tables and figures, symbols in Table 3 are to be used. The number of elements used in ABAQUS should be determined to obtain an acceptable solution for simply supported rectangular plates with point supports at the centre, as this type of problems is found in numerous literature items. Simply supported rectangular plates with a point support at centre are shown in Fig. 3. The results of QEM $(2 \times 2$ and $4 \times 4)$ are presented in Table 4 with other solutions for which different methods are applied. For the first five frequency parameters $\lambda$, all results are also in good agreement. Especially, the results of the finite strip element method used by Huang and Thambiratnam (2001) are strongly in agreement with QEM. If it is assumed that the first mode is 49.483 as it was taken from results of Huang's solution (Huang and Thambiratnam, 2001), Fig. 4 shows the change in the results from ABAQUS solution as a function of the number of elements on each side of the plate. It can be seen that the relative error according to Huang's results is approximately $0.03 \%$ for 100 elements on each side of the plate.

Table 3. Simplified support type symbols

\begin{tabular}{|c|c|}
\hline Symbol & Support types \\
\hline$\overline{\text { Null }}$ & Free \\
\hline "v|l||l||l||||||, & Fixed \\
\hline י - י & Simply \\
\hline$\circ$ & Point \\
\hline
\end{tabular}

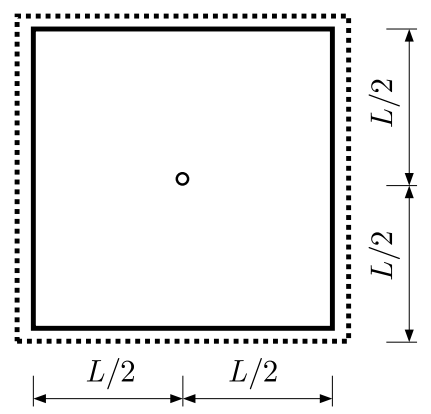

Fig. 3. Simply supported square plates with a point-support at center

As shown in Fig. 5, five boundary conditions and point support at the corner of the plate are considered. In Table 5, the results of Kim and Dickinson (1987) - orthogonal polynomial, Cheung and Zhou (2000) - static beam function, and Mizusawa and Kajita (1987) - finite spline, are presented. CFCF, CFSF, SFSF, CFFF, SFFF boundary conditions are considered and first five frequency parameters are presented. The natural frequencies are determined using both QEM and ABAQUS, and the obtained results are in good agreement with the analytical results reported in the literature.

For several cases, the results for plates with point supports are compared with other values given in the literature. As shown in Fig. 6, plates with different numbers of point supports at the interior and/or boundary are considered. All results obtained from ABAQUS and QEM solutions are presented in Table 6. Kato and Honma (1998), Kim and Dickinson (1987) used Rayleight-Ritz Method, Fan and Cheung (1984), Mizusawa and Kajita (1987) used Spline Finite Strip Element Method, Narita and Hodgkinson (2005) used Layerwise optimization method, Venkateswara et 
Table 4. Frequency parameters $\lambda$ of simply supported square plates with a point support at the center $\left(\lambda=\omega L^{2} \sqrt{\rho h / D}\right)$

\begin{tabular}{|l|c|c|c|c|c|}
\hline Method & $\lambda_{1}$ & $\lambda_{2}$ & $\lambda_{3}$ & $\lambda_{4}$ & $\lambda_{5}$ \\
\hline \hline Venkateswara et al. $(1973)$ & - & - & 52.62 & - & - \\
\hline Lee and Lee (1977) & - & - & 53.088 & - & - \\
\hline Leissa (1969) & 49.3 & - & - & - & - \\
\hline Saadatpour et al. $(2000)]$ & 49.348 & - & - & - & - \\
\hline Fan and Cheung (1984) & 49.35 & 49.35 & 52.78 & 78.96 & 98.71 \\
\hline Kim and Dickinson (1987)] & 49.348 & 49.348 & 53.170 & 78.959 & 98.696 \\
\hline Huang and Thambiratnam (2001b) & 49.348 & 49.351 & 52.667 & 78.959 & 98.711 \\
\hline Present (ABAQUS) & 49.362 & 49.362 & 52.643 & 78.975 & 98.784 \\
\hline Present (QEM, $2 \times 2)$ & 49.348 & 49.348 & 52.851 & 78.957 & 98.711 \\
\hline Present (QEM, 4×4) & 49.348 & 49.348 & 52.677 & 78.957 & 98.696 \\
\hline
\end{tabular}

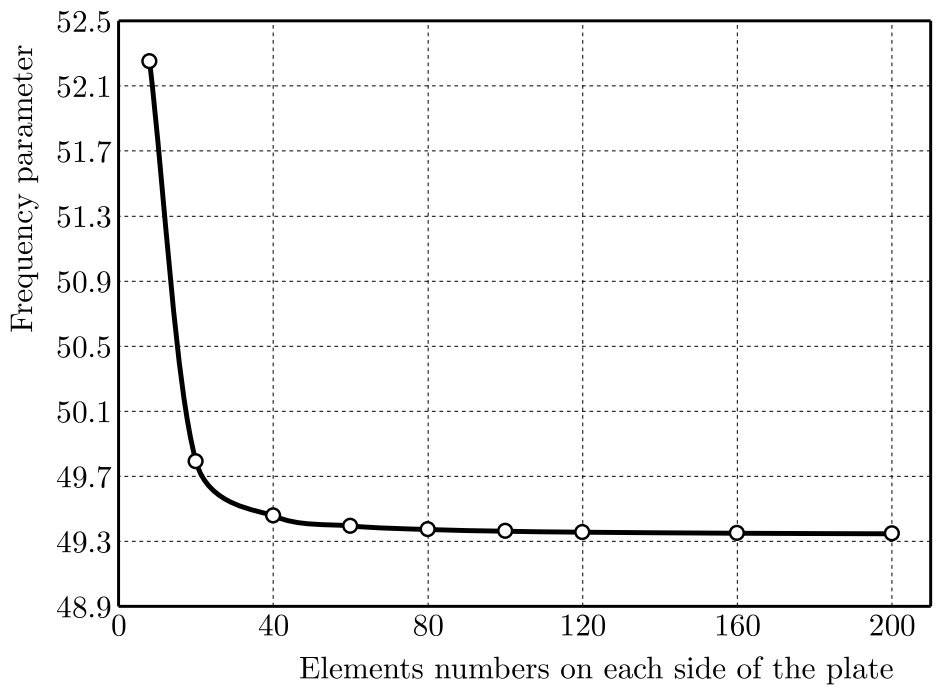

Fig. 4. First frequency parameters for simply supported square plate with a point support at center (ABAQUS solutions)

(a)

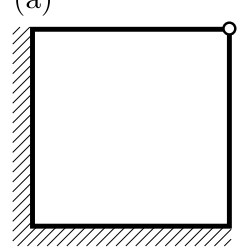

(b)

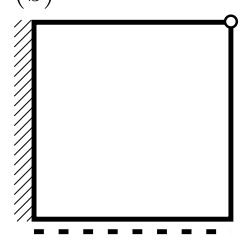

(c)

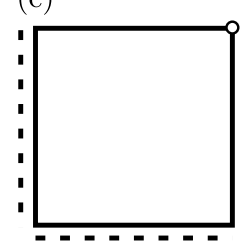

(d)

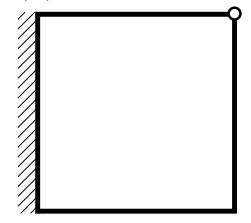

(e)

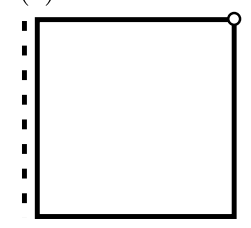

Fig. 5. Square plates with point supports at one corner for various boundary conditions

al. (1973) used Finite Element Method. Kocatürk et al. (2004) used the Lagrange Equation Method. The first five frequency parameters for eight different point supports situation are given in Table 6. It can be seen from Table 6, a very good agreement between QEM and those of Kato and Honma (1998), Kim and Dickinson (1987), Mizusawa and Kajita (1987), Narita and Hodgkinson (2005), Venkateswara et al. (1973), Kocatürk et al. (2004) are encountered.

Various point support topologies and four different types of boundary conditions are considered as shown in Table 7 . The minimum distances are $L / 4$ since four quadrature plate elements are used for solutions. Seven different situations are considered and the first five frequency parameters are calculated. SSSS, CCCC, SCSC and FCFS type of boundary conditions are selected. 
Table 5. Frequency parameters $\lambda$ of square plates with point supports at one corner for various boundary conditions $\left(\lambda=\omega L^{2} \sqrt{\rho h / D}\right)$

\begin{tabular}{|c|c|c|c|c|c|c|}
\hline Fig. & Method & $\lambda_{1}$ & $\lambda_{2}$ & $\lambda_{3}$ & $\lambda_{4}$ & $\lambda_{5}$ \\
\hline \multirow{6}{*}{$5 \mathrm{a}$} & Cheung and Zhou (1999) & 15.272 & 24.100 & 39.495 & 54.703 & 63.511 \\
\hline & Mizusawa and Kajita (1987) & 15.12 & 23.70 & 39.37 & 53.53 & 62.54 \\
\hline & Kim and Dickinson (1987) & 15.172 & 23.923 & 39.392 & 54.157 & 62.850 \\
\hline & Present (ABAQUS) & 15.166 & 23.905 & 39.394 & 54.105 & 62.742 \\
\hline & Present (QEM, $2 \times 2)$ & 15.169 & 23.915 & 39.389 & 54.112 & 62.718 \\
\hline & Present (QEM, $4 \times 4)$ & 15.166 & 23.906 & 39.388 & 54.094 & 62.708 \\
\hline \multirow{6}{*}{$5 b$} & Cheung and Zhou (1999) & 12.021 & 21.348 & 35.140 & 47.916 & 58.903 \\
\hline & Mizusawa and Kajita (1987) & 11.94 & 21.06 & 35.01 & 47.24 & 57.92 \\
\hline & Kim and Dickinson (1987) & 11.940 & 21.175 & 35.015 & 47.398 & 58.144 \\
\hline & Present (ABAQUS) & 11.939 & 21.167 & 35.018 & 47.399 & 58.096 \\
\hline & Present (QEM, $2 \times 2$ ) & 11.939 & 21.172 & 35.014 & 47.393 & 58.076 \\
\hline & Present (QEM, $4 \times 4)$ & 11.939 & 21.167 & 35.014 & 47.388 & 58.069 \\
\hline \multirow{6}{*}{$5 \mathrm{c}$} & Cheung and Zhou (1999) & 9.6801 & 17.496 & 30.713 & 44.178 & 51.873 \\
\hline & Mizusawa and Kajita (1987) & 9.608 & 17.32 & 30.60 & 43.65 & 51.04 \\
\hline & Kim and Dickinson (1987) & 9.6079 & 17.316 & 30.596 & 43.652 & 51.041 \\
\hline & Present (ABAQUS) & 9.6079 & 17.317 & 30.598 & 43.663 & 51.058 \\
\hline & Present (QEM, $2 \times 2)$ & 9.6079 & 17.316 & 30.596 & 43.652 & 51.036 \\
\hline & Present (QEM, $4 \times 4)$ & 9.6079 & 17.316 & 30.596 & 43.652 & 51.035 \\
\hline \multirow{5}{*}{$5 \mathrm{~d}$} & Cheung and Zhou (1999) & 5.3351 & 16.054 & 22.000 & 29.536 & 43.894 \\
\hline & Mizusawa and Kajita (1987) & 5.312 & 15.86 & 21.71 & 29.29 & 43.39 \\
\hline & Present (ABAQUS) & 5.3261 & 15.912 & 21.813 & 29.403 & 43.499 \\
\hline & Present (QEM, $2 \times 2)$ & 5.3277 & 15.915 & 21.817 & 29.407 & 43.497 \\
\hline & Present (QEM, $4 \times 4)$ & 5.3268 & 15.912 & 21.812 & 29.403 & 43.494 \\
\hline \multirow{5}{*}{$5 \mathrm{e}$} & Cheung and Zhou (1999) & 3.3395 & 12.033 & 17.419 & 25.886 & 38.982 \\
\hline & Mizusawa and Kajita (1987) & 3.336 & 11.93 & 17.29 & 25.68 & 38.56 \\
\hline & Present (ABAQUS) & 3.3357 & 11.927 & 17.293 & 25.681 & 38.561 \\
\hline & Present (QEM, $2 \times 2)$ & 3.3361 & 11.927 & 17.293 & 25.680 & 38.555 \\
\hline & Present (QEM, $4 \times 4)$ & 3.3361 & 11.927 & 17.293 & 25.679 & 38.555 \\
\hline
\end{tabular}

(a)

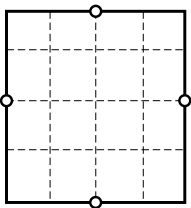

(e)

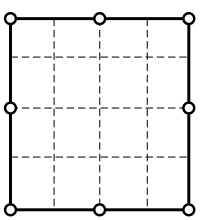

(b)

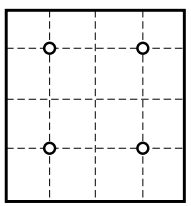

(f)

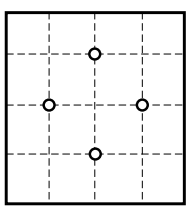

(c)

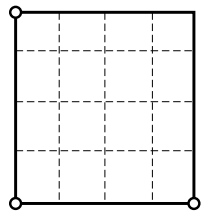

(g)

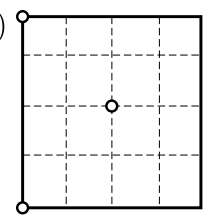

(d)

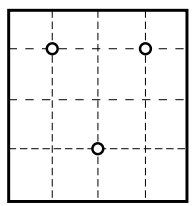

(h)

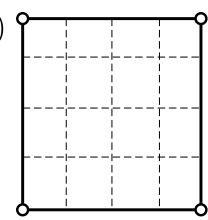

Fig. 6. Square plates with point supports

Besides, the results of point supported free plates are given in Table 8. The first five frequency parameters are presented for point supports on the interior and/or boundary of plates. The differences between the results of QEM and ABAQUS solutions are approximately $0.1 \%$ 
Table 6. Comparison of frequency parameters $\lambda$ of square plates with point supports $\left(\lambda=\omega L^{2} \sqrt{\rho h / D}\right)$

\begin{tabular}{|c|c|c|c|c|c|c|}
\hline Fig. & Method & $\lambda_{1}$ & $\lambda_{2}$ & $\lambda_{3}$ & $\lambda_{4}$ & $\lambda_{5}$ \\
\hline \multirow{6}{*}{$6 \mathrm{a}$} & Kato and Honma (1998) & 13.51 & 18.03 & $\overline{19.05}$ & 19.05 & 27.26 \\
\hline & Kim and Dickinson (1987) & 13.47 & 18.03 & 18.93 & 18.93 & 27.05 \\
\hline & Fan and Cheung (1984) & 13.47 & 17.85 & 18.79 & 18.79 & 26.92 \\
\hline & Narita and Hodgkinson (2005) & 13.47 & 18.14 & 19.02 & 19.02 & $\overline{-}$ \\
\hline & Present (ABAQUS) & 13.468 & 17.835 & 18.780 & 18.780 & 26.910 \\
\hline & Present (QEM) & 13.468 & 17.841 & 18.786 & 18.786 & 26.913 \\
\hline \multirow{3}{*}{$6 \mathrm{~b}$} & Narita and Hodgkinson (2005) & 19.60 & 23.40 & 33.17 & 33.17 & - \\
\hline & Present (ABAQUS) & 19.598 & 23.380 & 32.580 & 32.580 & 34.985 \\
\hline & Present (QEM) & 19.596 & 23.378 & 32.597 & 32.597 & 35.013 \\
\hline \multirow{3}{*}{$6 c$} & Narita and Hodgkinson (2005) & 3.299 & 9.894 & 15.77 & 19.60 & - \\
\hline & Present (ABAQUS) & 3.298 & 9.893 & 15.769 & 19.598 & 26.618 \\
\hline & Present (QEM) & 3.298 & 9.893 & 15.770 & 19.596 & 26.616 \\
\hline \multirow{3}{*}{$6 \mathrm{~d}$} & Narita and Hodgkinson (2005) & 9.512 & 14.78 & 21.34 & 29.09 & - \\
\hline & Present (ABAQUS) & 9.486 & 14.659 & 21.309 & 28.841 & 33.586 \\
\hline & Present (QEM) & 9.487 & 14.662 & 21.307 & 28.847 & 33.604 \\
\hline \multirow{5}{*}{$6 e$} & Kato and Honma (1998) & 18.03 & 35.62 & 35.62 & 38.68 & 61.06 \\
\hline & Kim and Dickinson (1987) & 18.03 & 35.17 & 35.17 & 38.43 & 60.58 \\
\hline & Fan and Cheung (1984) & 17.85 & 34.89 & 34.89 & 38.43 & 60.12 \\
\hline & Present (ABAQUS) & 17.837 & 34.884 & 34.884 & 38.440 & 60.101 \\
\hline & Present (QEM) & 17.843 & 34.882 & 34.882 & 38.432 & 60.086 \\
\hline \multirow{3}{*}{$6 f$} & Narita and Hodgkinson (2005) & 13.47 & 17.09 & 18.65 & 18.65 & - \\
\hline & Present (ABAQUS) & 13.468 & 17.029 & 18.275 & 18.275 & 39.185 \\
\hline & Present (QEM) & 13.468 & 17.030 & 18.284 & 18.284 & 39.215 \\
\hline \multirow{3}{*}{$6 \mathrm{~g}$} & Narita and Hodgkinson (2005) & 6.641 & 6.736 & 19.60 & 19.75 & - \\
\hline & Present (ABAQUS) & 6.638 & 6.700 & 19.489 & 19.598 & 24.639 \\
\hline & Present (QEM) & 6.639 & 6.701 & 19.495 & 19.596 & 24.639 \\
\hline \multirow{7}{*}{$6 \mathrm{~h}$} & Narita and Hodgkinson (2005) & 7.112 & 15.77 & 15.77 & 16.90 & - \\
\hline & Cheung and Zhou (1999) & 7.136 & 15.800 & 15.805 & 19.710 & 38.710 \\
\hline & Mizusawa and Kajita (1987) & 7.111 & 15.77 & 15.77 & 19.60 & 38.43 \\
\hline & Kocatürk et al. (2004) & 7.1109 & $\overline{-}$ & - & 19.596 & - \\
\hline & Venkateswara et al. (1973) & 7.1109 & - & - & 19.596 & - \\
\hline & Present (ABAQUS) & 7.1112 & 15.769 & 15.769 & 19.598 & 38.440 \\
\hline & Present (QEM) & 7.1109 & 15.770 & 15.770 & 19.596 & 38.432 \\
\hline
\end{tabular}

\section{Conclusions}

The Quadrature Element Method is applied to analyze free vibration of point supported rectangular plates having different boundary conditions and various point topologies. The results are compared to other approximation methods. A very good agreement is observed with the data published in literature. A 25-node plate element is easier to process with commercial software. It is possible to apply the Quadrature Element Method to plates having more complex shapes and to obtain a better accuracy by means of joining plate elements side by side along the $x$ and $y$ directions. 
Table 7. Frequency parameters $\lambda$ of square plates with point supports for four boundary conditions $\left(\lambda=\omega L^{2} \sqrt{\rho h / D}\right)$

\begin{tabular}{|c|c|c|c|c|c|c|c|c|c|}
\hline \multirow{2}{*}{$\begin{array}{l}\text { Support } \\
\text { Position }\end{array}$} & \multirow{2}{*}{ Mod } & \multicolumn{2}{|c|}{ S-S-S-S } & \multicolumn{2}{|c|}{ C-C-C-C } & \multicolumn{2}{|c|}{ C-S-C-S } & \multicolumn{2}{|c|}{ F-C-F-S } \\
\hline & & QEM & ABAQUS & QEM & ABAQUS & QEM & ABAQUS & QEM & ABAQUS \\
\hline & $\lambda_{1}$ & 167.78 & 168.08 & 187.97 & 188.10 & 170.35 & 170.64 & 48.538 & 48.515 \\
\hline & $\overline{\lambda_{2}}$ & 167.78 & 168.08 & 187.97 & 188.10 & 184.95 & 184.97 & 50.128 & 50.086 \\
\hline & $\overline{\lambda_{3}}$ & 182.71 & 182.74 & 207.96 & 207.92 & 185.99 & 186.13 & 82.215 & 82.172 \\
\hline & $\lambda_{4}$ & 182.71 & 182.74 & 215.98 & 215.71 & 205.14 & 205.04 & 82.772 & 82.712 \\
\hline & $\overline{\lambda_{5}}$ & 197.39 & 197.65 & 242.16 & 242.60 & 206.70 & 206.96 & 133.17 & 133.19 \\
\hline & $\lambda_{1}$ & 49.348 & 49.362 & 73.394 & 73.437 & 60.807 & 60.829 & 26.227 & 26.222 \\
\hline & $\overline{\lambda_{2}}$ & 62.106 & 62.071 & 86.985 & 86.931 & \begin{tabular}{|l|}
73.233 \\
\end{tabular} & 73.194 & 33.799 & 33.787 \\
\hline & $\lambda_{3}$ & 91.269 & 91.232 & 105.57 & 105.55 & 100.02 & 99.999 & 61.801 & 61.796 \\
\hline & $\overline{\lambda_{4}}$ & 98.696 & 98.784 & 131.58 & 131.76 & 115.97 & 116.083 & 66.573 & 66.574 \\
\hline & $\lambda_{5}$ & 128.30 & 128.38 & 151.28 & 151.16 & 141.12 & 140.983 & 77.381 & 77.385 \\
\hline & $\lambda_{1}$ & 78.957 & 78.975 & 108.22 & 108.27 & 94.586 & 94.625 & 42.012 & 41.987 \\
\hline & $\lambda_{2}$ & 91.269 & 91.228 & 121.28 & 121.25 & 104.68 & 104.66 & 42.899 & 42.859 \\
\hline & $\lambda_{3}$ & 91.269 & 91.228 & 121.28 & 121.24 & 110.16 & 110.10 & 58.187 & 58.196 \\
\hline & $\overline{\lambda_{4}}$ & 101.69 & 101.61 & 139.20 & 139.12 & 120.37 & 120.30 & 61.114 & 61.109 \\
\hline & $\lambda_{5}$ & 167.78 & 168.08 & 204.49 & 204.88 & 170.35 & 170.64 & 99.245 & 99.301 \\
\hline & $\lambda_{1}$ & 67.760 & 67.759 & 74.089 & 74.075 & 71.703 & 71.701 & 38.804 & 38.798 \\
\hline & $\lambda_{2}$ & 91.269 & 91.232 & 105.57 & 105.55 & 104.68 & 104.66 & 48.538 & 48.515 \\
\hline & $\overline{\lambda_{3}}$ & 131.52 & 131.51 & 162.74 & 162.81 & 162.17 & 162.25 & 77.124 & 77.123 \\
\hline & $\lambda_{4}$ & 167.78 & 168.08 & 187.97 & 188.10 & 170.33 & 170.64 & 80.303 & 80.260 \\
\hline & $\lambda_{5}$ & 167.78 & 168.08 & 207.05 & 207.49 & 193.81 & 193.78 & 82.215 & 82.172 \\
\hline & $\lambda_{1}$ & 52.677 & 52.644 & 55.185 & 55.150 & 53.966 & 53.931 & 38.203 & 38.193 \\
\hline & $\overline{\lambda_{2}}$ & 91.269 & 91.232 & 105.57 & 105.55 & 92.350 & 92.298 & 41.679 & 41.647 \\
\hline & $\lambda_{3}$ & 91.269 & 91.232 & 105.57 & 105.55 & 104.68 & 104.66 & 52.440 & 52.410 \\
\hline & $\lambda_{4}$ & 98.696 & 98.784 & 131.58 & 131.76 & 110.61 & 110.70 & 77.139 & 77.137 \\
\hline & $\lambda_{5}$ & 146.83 & 146.80 & 180.45 & 180.55 & 168.11 & 168.16 & 79.983 & 79.957 \\
\hline & $\lambda_{1}$ & 91.269 & 91.232 & 105.57 & 105.55 & 92.350 & 92.298 & 39.993 & 39.972 \\
\hline & $\lambda_{2}$ & 91.269 & 91.232 & 105.57 & 105.55 & 103.43 & 103.33 & 41.679 & 41.647 \\
\hline & $\lambda_{3}$ & 98.696 & 98.784 & 116.08 & 115.81 & 104.68 & 104.66 & 75.963 & 75.960 \\
\hline & $\lambda_{4}$ & 104.81 & 104.58 & 131.58 & 131.76 & 120.48 & 120.44 & 79.983 & 79.957 \\
\hline & $\lambda_{5}$ & 167.78 & 168.07 & 207.05 & 207.49 & 170.35 & 170.64 & 96.537 & 96.564 \\
\hline
\end{tabular}

\section{References}

1. Bapat A.V., Suryanarayan S., 1989, Flexibility function approach to vibration analysis of rectangular plates with arbitrary multiple point supports on the edges, Journal of Sound and Vibration, 128, 209-233

2. Bellman R., Casti J., 1971, Differential quadrature and long-term integration, Journal of Mathematical Analysis and Applications, 34, 235-238

3. Chen C.-N., 2004, DQEM and DQFDM irregular elements for analyses of 2-D heat conduction in orthotropic media, Applied Mathematical Modeling, 28, 7, 617-638

4. Chen W.L., Striz A.G., Bert C.W., 2000, High-accuracy plane stress and plate elements in the quadrature element method, International Journal of Solids and Structures, 37, 627-647

5. Cheung Y.K., Zhou D., 1999, Free vibration of rectangular composite plates with point-supports using static beam functions, Composite Structures, 44, 2, 145-154 
Table 8. Frequency parameters of free square plates with point supports $\left(\lambda=\omega L^{2} \sqrt{\rho h / D}\right)$

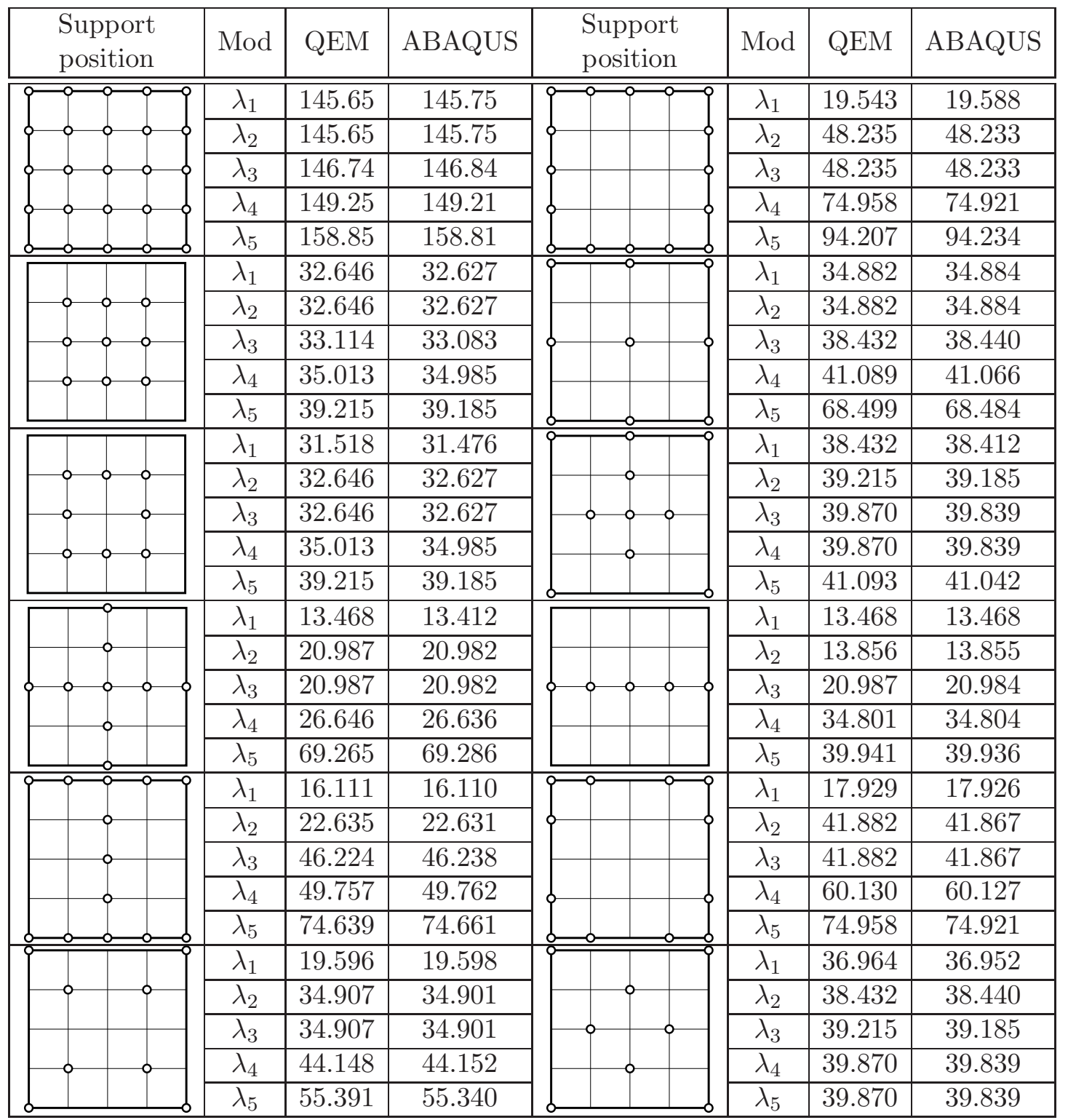

6. Cheung Y.K., Zhou D., 2000, Free vibration of thick, layered rectangular plates with point supports by finite layer method, International Journal of Solids and Structures, 37, 10, 1483-1499

7. Cox H.L., Boxer J., 1960, Vibration of rectangular plates point-supported at the corners, Aeronautical Quarterly, 11, 41-50

8. Damle S.K., Feeser L.J., 1972, Vibration of four point supported plates by a finite element method, Journal of the Aeronautical Society of India, 24, 375-377

9. FAn S.C., Cheung Y.K., 1984, Flexural free vibrations of rectangular plates with complex support conditions, Journal of Sound and Vibration, 93, 81-94

10. Franciosi C., Tomasiello S., 2004, A modified quadrature element method to perform static analysis of structures, International Journal of Mechanical Sciences, 46, 6, 945-959

11. Gorman D.J., 1991, Analytical and experimental study of vibrating rectangular plates on rigid point supports, AIAA Journal, 29, 8, 38-44

12. Gu H.Z., Wang X.W., 1997, On the free vibration analysis of circular plates with stepped thickness over a concentric region by the quadrature element method, Journal of Sound and Vibration, 202, $452-459$ 
13. Gutierrez R.H., Laura P.A.A., 1995, Analysis of vibrating, thin, rectangular plates with point-supports by the method of differential quadrature, Ocean Engineering, 22, 101-103

14. HAN J.B., LIEW K.M., 1996, The differential quadrature element method (DQEM) for axisymmetric bending of thick circular plates, Proceedings of the 3rd Asian-Pacific Conference on Computational Mechanics, 2363-2368

15. Huang M.H., Thambiratnam D.P., 2001a, Analysis of plate resting on elastic supports and elastic foundation by finite strip method, Computers and Structures, 79, 29-30, 2547-2557

16. Huang M.H., Thambiratnam D.P., 2001b, Free vibration analysis of rectangular plates on elastic intermediate supports, Journal of Sound and Vibration, 240, 3 567-580

17. Kato Y., Honma T., 1998, The Rayleigh-Ritz solution to estimate vibration characteristics of building floors, Journal of Sound and Vibration, 211, 2, 195-206

18. Kerstens J.G.M., 1979, Vibration of a rectangular plate supported at an arbitrary number of points, Journal of Sound and Vibration, 65, 493-504

19. Kim C.S., DiCKInson S.M., 1987, Flexural vibration of rectangular plates with point supports, Journal of Sound and Vibration, 117, 249-261

20. Kitipornchai S., Xiang Y., Liew K.M., 1994, Vibration of analysis of corner supported Mindlin plates of arbitrary shape using the Lagrange multiplier method, Journal of Sound and Vibration, 173, $457-470$

21. Kocatürk T., Sezer S., Demir C., 2004, Determination of the steady state response of viscoelastically point-supported rectangular specially orthotropic plates with added concentrated masses, Journal of Sound and Vibration, 278, 4-5, 789-806

22. LEE L.T., LEE D.C., 1997, Free vibration of rectangular plates on elastic point supports with the application of a new type of admissible function, Computer and Structures, 65, 2, 149-156

23. LeIssA A.W., 1969, Vibration of plates, NASA Sp160, U.S. Government Printing Office

24. Leissa A.W., 1973, The free vibration of rectangular plates, Journal of Sound and Vibration, 31 , 3, 257-293

25. LiEw K.M., LAM K.Y., 1994, Effects of arbitrarily distributed elastic point constraints on vibrational behavior of rectangular plates, Journal of Sound and Vibration, 174, 1, 23-36

26. Liew K.M., Xiang Y., Kitipornchai S., 1994, Vibration of Mindlin plates on point supports using constraint functions, Journal of Engineering Mechanics, 120, 499-513

27. Liu F.-L., LiEw K.M., 1998, Static analysis of Reissner-Mindlin plates by differential quadrature element method, ASME Journal of Applied Mechanics, 65, 705-710

28. LiU F.-L., LiEw K.M., 1999a, Differential quadrature element method: a new approach for free vibration analysis of polar Mindlin plates having discontinuities, Computer Methods in Applied Mechanics and Engineering, 179, 3-4, 407-423

29. LiU F.-L., LiEw K.M., 1999b, Differential quadrature element method for static analysis of Reissner-Mindlin polar plates, International Journal of Solids and Structures, 36, 5101-5123

30. LiU F.-L., 2000, Rectangular thick plates on Winkler foundation: differential quadrature element solutions, International Journal of Solids and Structures, 37, 12, 1743-1763

31. Mizusawa T., Kajita T., 1987, Vibration of skew plates resting on point supports, Journal of Sound and Vibration, 115, 243-251

32. Narita Y., Hodgkinson J.M., 2005, Layerwise optimisation for maximizing the fundamental frequencies of point-supported rectangular laminated composite plates, Composite Structures, 69, $2,127-135$

33. QuAn J.R., Chang C.T., 1989, New insights in solving distributed system equations by the quadrature method. I - Analysis, Computers and Chemical Engineering, 13, 7, 779-788 
34. Rui L., Bo W., Gang L., Bin T., 2016, Hamiltonian system-based analytic modeling of the free rectangular thin plates' free vibration, Applied Mathematical Modelling, 40, 2, 984-992

35. Rui L., Bo W., Gang L., Jiahui D., Xiaoting A., 2015, Analytic free vibration solutions of rectangular thin plates point-supported at a corner, International Journal of Mechanical Sciences, 96-97, 199-205

36. Saadatpour M.M., Azhari M., Bradford M.A., 2000, Vibration analysis of simply supported plates of general shape with internal point and line supports using the Galerkin method, Engineering Structures, 22, 9, 1180-1188

37. Striz A.G., Chen W., Bert C.W., 1994, Static analysis of structures by the quadrature element method (QEM), International Journal of Solids and Structures, 31, 20, 2807-2818

38. Venkateswara R.G., Raju I.S., Murty G.K., 1073, Vibration of rectangular plates with mixed boundary conditions, Journal of Sound and Vibration, 30, 257-260

39. WANG X.W., Gu H.Z., 1997, Static analysis of frame structure by the differential quadrature element method, International Journal of Numerical Methods in Engineering, 40, 759-772

40. Zhao Y.B., Wei G.W., XiAng Y., 2002, Plate vibration under irregular internal supports, International Journal of Solids and Structures, 39, 5, 361-1383

41. ZHOU D., 2002, Vibrations of point-supported rectangular plates with variable thickness using a set of static tapered beam functions, International Journal of Mechanical Sciences, 44, 1, 149-164

Manuscript received June 4, 2016; accepted for print April 10, 2017 